\title{
Treelike Interactions and Fast Scrambling with Cold Atoms
}

\author{
Gregory Bentsen, ${ }^{1}$ Tomohiro Hashizume,${ }^{2}$ Anton S. Buyskikh, ${ }^{2}$ Emily \\ J. Davis, ${ }^{1}$ Andrew J. Daley, ${ }^{2}$ Steven S. Gubser, ${ }^{3}$ and Monika Schleier-Smith ${ }^{1}$ \\ ${ }^{1}$ Department of Physics, Stanford University, Stanford, CA 94305, USA \\ ${ }^{2}$ Department of Physics and SUPA, University of Strathclyde, Glasgow G4 0NG, UK \\ ${ }^{3}$ Department of Physics, Princeton University, Princeton, NJ 08544, USA
}

(Dated: October 26, 2019)

\begin{abstract}
We propose an experimentally realizable quantum spin model that exhibits fast scrambling, based on non-local interactions which couple sites whose separation is a power of 2. By controlling the relative strengths of deterministic, non-random couplings, we can continuously tune from the linear geometry of a nearest-neighbor spin chain to an ultrametric geometry in which the effective distance between spins is governed by their positions on a tree graph. The transition in geometry can be observed in quench dynamics, and is furthermore manifest in calculations of the entanglement entropy. Between the linear and treelike regimes, we find a peak in entanglement and exponentially fast spreading of quantum information across the system. Our proposed implementation, harnessing photon-mediated interactions among cold atoms in an optical cavity, offers a test case for experimentally observing the emergent geometry of a quantum many-body system.
\end{abstract}

The fast scrambling conjecture -inspired by studies of the black-hole information problem - predicts a lower bound on the time for information to spread from one to all degrees of freedom of an $N$-body quantum system, scaling as $t_{*} \propto \log (N)[1,2]$. Fast scrambling is conceptually important as a putative signature of the quantum physics of black holes [3]. It is also of practical importance because a fast scrambler is an efficient quantum encoder, capable of quickly entangling quantum information across many physical qubits [4]. While information scrambling has been probed in several pioneering experiments [5-8], observing fast scrambling remains an outstanding challenge.

In typical quantum systems found in nature, fast scrambling is precluded by the locality of interactions. In the presence of only short-range interactions, information propagation is bounded by a linear "light cone" [9-12] (Fig. 1a), such that the scrambling time is at least $t_{*} \propto N$. Even with long-range power-law interactions [13-15], the time for quantum correlations to propagate scales polynomially with distance $[14,15]$ and not logarithmically. Potential work-arounds include engineering interactions among spatially overlapped modes $[16-18]$ or using light to mediate effectively non-local interactions $[19,20]$. Proposals to date have focused on emulating solvable models for fast scrambling featuring random allto-all couplings [16-18, 20].

In this Letter, we propose an experimentally realizable quantum spin model featuring a sparse, deterministic graph of non-local interactions that enables fast scrambling. A modest generalization of the simplest model provides a smooth interpolation between two radically different notions of geometry: linear or treelike, as shown in Fig. 1b. Our simplest model is, in a certain sense, exactly between these two limits. Instead of exhibiting extended spatial geometry, it is fully characterized by a coupling graph whose diameter grows logarithmically

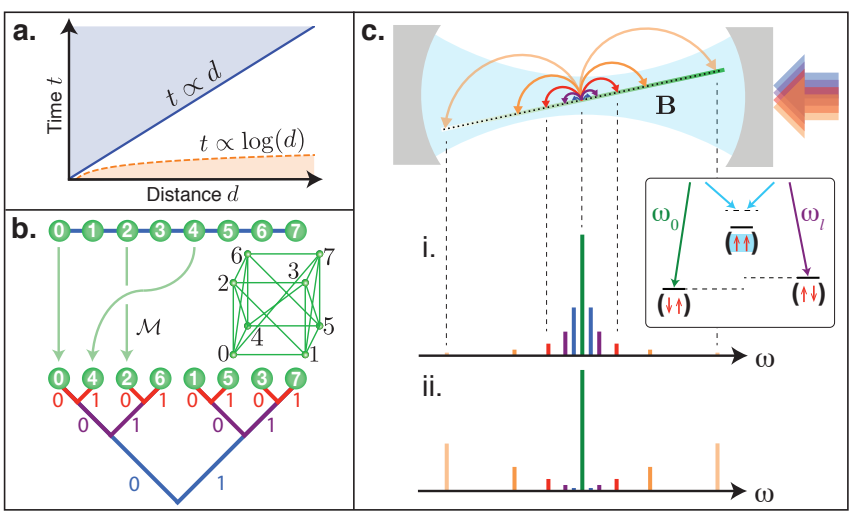

FIG. 1. Nonlocal spin models with cold atoms. (a) Linear light cone vs. fast scrambling. (b) Spins (green) coupled locally along a chain of length $N=8$ or non-locally according to a tree of depth $n=\log _{2}(N)$. Green cube shows full pattern of couplings specified in Eq. (2). (c) Scheme for controlling the graph of interactions mediated by a cavity via the spectrum of a drive field and the gradient of a magnetic field B. Depending whether short-range (blue) or long-range (orange) couplings dominate, the graph resembles either the linear chain (i) or the tree (ii).

with the number of sites, resulting in exponentially fast spreading of perturbations that decreases to power-law spreading as we tune away from the original model. Thus, loosely speaking, we see the exponential spread of perturbations as a form of criticality encountered between two incompatible geometric notions of locality (Fig. 1b).

In studying this transition in geometry, we are also motivated by holography, in particular the recently proposed $p$-adic version $[21,22]$ of the anti-de Sitter / Conformal Field Theory correspondence (AdS/CFT). Here, the role of the gravitational bulk is played by the BruhatTits tree, which is an infinite regular tree with $p+1$ edges leading into each vertex, similar to Fig. $1 \mathrm{~b}$ for $p=2$. Past 
works have suggested that diffusion on the Bruhat-Tits tree can be used to understand aspects of fast scrambling near black hole horizons [23, 24].

The models we consider here are inspired by an elegant proposal [25] for engineering translation-invariant long-range interactions by encoding spins in cold atoms coupled to light in a waveguide or cavity [26-37]. This scheme can generate Hamiltonians of the form

$$
H=\frac{1}{2 S} \sum_{i, j} J(i-j) S_{i}^{+} S_{j}^{-},
$$

where $i, j=1, \ldots, N$, and $\mathbf{S}_{i}$ is a spin- $S$ operator representing either an individual atom or the collective spin of a small atomic ensemble at site $i$. We consider a sparse graph of couplings between sites separated by powers of two,

$$
J(i-j)= \begin{cases}J_{s} 2^{\ell s} & \text { when }|i-j|=2^{\ell}, \ell=0,1,2,3 \ldots \\ 0 & \text { otherwise, }\end{cases}
$$

where by tuning the exponent $s$ from $-\infty$ to $+\infty$ we interpolate between the linear and treelike limits. The simplest choice $s=0$, where all non-zero couplings are equal, permits a perturbation to spread exponentially because the number of pairwise interactions required to get from site $i$ to site $j$ is bounded above by the number of binary digits in $i$ and $j$ that differ.

The couplings in Eq. (2) can be generated by a pair of Raman processes wherein one atom flips its spin by virtually scattering a photon from a control field into a cavity, and a second atom rescatters this photon such that its spin flops [31]. In a magnetic field gradient [25], the energy cost of a flip-flop is proportional to the distance $d=i-j$ between spins. Thus, inducing resonant flip-flops at distance $|d|=2^{\ell}$ requires modulating the control field at a frequency $\omega_{\ell} \propto d$. More generally, weakly modulating the control field at multiple frequencies $\omega_{\ell}$ for $\ell=0,1,2,3, \ldots$ produces a set of sidebands whose amplitudes dictate the hopping amplitudes $J(d)$ (Fig. 1).

Below, we take $N$ a power of 2 and assume periodic boundary conditions unless otherwise stated, letting $|x|$ denote the minimum value of $\sqrt{(x+q N)^{2}}$ over all integers $q$. We normalize the couplings in Eq. (2) such that the largest is always a constant $J_{0}$, letting $J_{s}=J_{0}$ for $s \leq 0$ and $J_{s}=J_{0}(N / 2)^{-s}$ for $s>0$.

The key features of our spin model are evident already in the spreading of a single initially localized spin excitation (Fig. 2a), which is governed by the magnon dispersion relation (Fig. 2b)

$$
E(k)=2 J_{s} \sum_{\ell=0}^{\log _{2}(N / 2)} 2^{\ell s} \cos \left(2^{\ell} k\right),
$$

where $k \in[0,2 \pi)$ is the wavenumber. Intriguingly, as $N$ increases, the dispersion relation shows fractal behavior

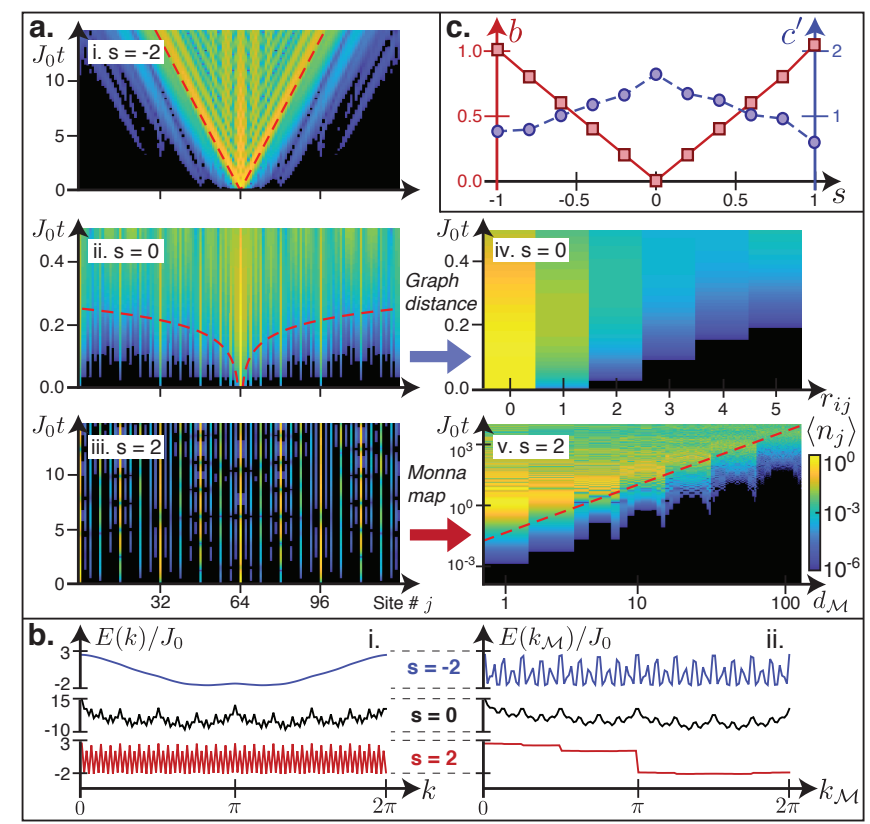

FIG. 2. Dynamics in the single-magnon sector. (a) Occupation $\left\langle n_{j}(t)\right\rangle$ for $N=128$ sites initialized with a single excitation on site $N / 2$ with (i) $s=-2$, (ii) $s=0$, or (iii) $s=2$; slowest-growing occupations are bounded from above by dashed red lines. (iv) Occupation $\left\langle n_{j}(t)\right\rangle$ at $s=0$ for $N=1024$, vs. graph distance $r_{i j}$ from initial site $i$. (v) Reemergence of light cone at $s=2$ after rearranging $N=128$ sites according to Monna map $\mathcal{M}$. (b) Magnon dispersion relation for $N=128$ sites arranged according to either (i) physical location or (ii) Monna-mapped order for $s=-2$ (blue), $s=0$ (black), and $s=2$ (red). (c) Breakdown of polynomial light cone: polynomial exponent $b$ (red) and logarithmic exponent $c^{\prime}$ (blue) versus $s$.

converging to a Weierstrass function for $0<s<1$ [38]. For arbitrary $s$, Eq. (3) allows us to analytically compute the single-magnon dynamics as shown in Fig. 2a, where we introduce a single excitation on site $i=N / 2$ and plot the mean occupation $\left\langle n_{j}(t)\right\rangle$ as a function of site number $j$ and time $t$.

The single-particle dynamics reveals the geometry of the interaction graph and its dependence on the exponent $s$. When $s$ is large and negative, the excitation spreads ballistically, as expected for a nearest-neighbor spin chain, producing the linear light cone in Fig. 2a(i). By contrast, for $s>0$, where interactions grow with physical distance, the excitation jumps discontinuously between distant sites (Fig. 2a(iii)). Rather than interpreting the apparent absence of a light cone for $s>0$ as the absence of locality, we argue that a new version of locality emerges based on the 2-adic norm $|x|_{2}=2^{-v(x)}$, where $2^{v(x)}$ is the largest power of 2 that divides $x$. The distance $|i-j|_{2}$ between sites $i$ and $j$ is called ultrametric because the distance of the sum of two steps is never greater than the larger of the two steps' distance; by contrast the usual distance $|i-j|$ is called Archimedean 
because many small steps can be combined into a large jump.

We can understand the 2-adic norm as a tree-like measure of distance because $|i-j|_{2}=2^{d_{\text {tree }}(i, j) / 2} / N$, where $d_{\text {tree }}(i, j)$ is the number of edges between sites $i$ and $j$ along the regular tree in Fig. 1b [39]. The leaves are numbered in order of increasing $\mathcal{M}(i)$, where the discrete Monna map $\mathcal{M}$ reverses the bit order in the site number. For example, for $N=8$ sites, $\mathcal{M}(1)=4$ because in binary, $\mathcal{M}\left(001_{2}\right)=100_{2}$. Noting that $N k / 2 \pi$ is an integer, we may likewise define a Monna-mapped wavenumber $k_{\mathcal{M}}$ by

$$
N \frac{k_{\mathcal{M}}}{2 \pi}=\mathcal{M}\left(N \frac{k}{2 \pi}\right)
$$

For large positive $s$, we rearrange the spins according to the Monna map and find that a light cone reappears (Fig. 2a(v)) and the dispersion relation is smoothed out (Fig. 2b), corroborating the transformation to the treelike geometry defined by the 2 -adic norm.

The radical difference between Archimedean and ultrametric geometry raises the question of what happens near $s=0$, where short- and long-range couplings are equally strong. Here, it is useful to think of the sites as arranged on a hypercube [40], where each site number in binary specifies a corner. The coupling graph consists of the edges of the hypercube plus some diagonals, as shown in Fig. 1b for $N=8$ sites. For any $N$, the graph distance $r_{i j}$ counts the minimum number of edges required to connect sites $i$ and $j$, and is at most $\left\lceil\frac{1}{2} \log _{2}(N)\right\rceil$, where $\lceil x\rceil$ denotes the smallest integer greater than or equal to $x$.

We expect the logarithmic diameter of the interaction graph at $s=0$ to enable a localized perturbation to spread over the entire system in a logarithmic time $t \propto \log (N)$. As a first test, in Fig. 2a we examine singlemagnon transport as a function of both physical distance $d=|i-j|$ and graph distance $r_{i j}$. We observe spin transport on a timescale that is roughly linear in graph distance (Fig. 2a(iv)), and thus at most logarithmic in physical distance $d$ and system size $N$.

To permit this logarithmic timescale, the polynomial light cone in Fig. 2a(i) must break down as $s$ approaches 0 . To examine this breakdown, we evaluate the time $t_{\epsilon}$ for the magnon occupation at a distance $d$ from the initial site to reach a threshold value $\left\langle n_{i+d}\right\rangle=\epsilon=1 / N^{2}$. For arbitrary $s$, we may always bound $t_{\epsilon}$ from below by a polynomial of the form $a\left[d_{(\mathcal{M})}\right]^{b} \leq t_{\epsilon}$ that depends on physical distance $d$ for $s \leq 0$ or Monna distance $d_{\mathcal{M}} \equiv$ $\mathcal{M}(d)$ for $s>0$, with non-negative constants $a, b$. We determine the dependence of the exponent $b$ on $s$ from a fit to the points of fastest growth [41] in Fig. 2a, finding a direct proportionality $b \propto|s|$ (red points in Fig. 2c). The vanishing exponent $b$ at $s=0$ signifies the breakdown of the polynomial light cone.

Furthermore, we verify that even the slowest-growing occupations take only a logarithmic time to reach the
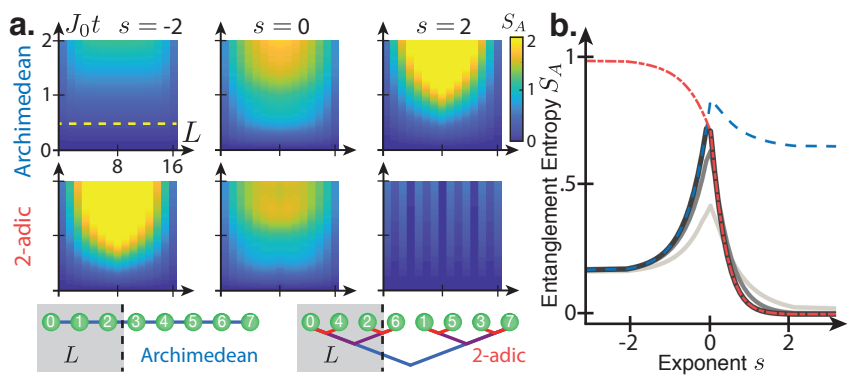

FIG. 3. Growth of entanglement after a quench, for an initial product state of $N=16$ sites with $S=1 / 2$ oriented along $\hat{\mathbf{x}}$. (a) Entanglement entropy $S_{A}$ vs. partition size $L$ and time $t$, for Archimedean (top) or 2-adic (bottom) partitions. (b) Minimum entanglement vs. $s$ over all possible partitions of size $L=1,2,4,8$ (light to dark solid curves), compared with entanglement for Archimedean (blue dashed) and 2-adic (red dot-dashed) partitions.

threshold $\epsilon$. For arbitrary $s$, the time $t_{\epsilon}$ is bounded above by $t_{\epsilon} \leq a^{\prime}\left[d_{(\mathcal{M})}\right]^{b^{\prime}}\left[\log d_{(\mathcal{M})}\right]^{c^{\prime}}$, with non-negative constants $a^{\prime}, b^{\prime}, c^{\prime}$ (dashed red lines in Fig. 2a) [41]. Here, as $s \rightarrow 0$ the polynomial exponent $b^{\prime}$ again disappears, but the logarithmic exponent $c^{\prime}$ remains (blue points in Fig. 2c). For both the fastest- and slowest-growing occupations we observe an approximate symmetry about $s=0$ that suggests a duality between the Archimedean and non-Archimedean regimes [44].

While the spin dynamics provides strong evidence for the underlying geometry of the model as a function of $s$, the structure of entanglement provides an even sharper proxy for locality. We analyze the growth of entanglement for a system initialized in a product state of spins polarized along $\hat{\mathbf{x}}$. Figure 3a shows the entanglement entropy $S_{A}$ of a subsystem $A$ as a function of its size and time, with the spins partitioned either according to their physical position (top) or to their Monna-mapped ordering (bottom). In either of the two limits where $|s|$ is large, there is a natural way of partitioning the system such that entanglement remains low, as summarized in Fig. 3b: for $s<0$, the cut must be between nearest neighbors in the linear chain (blue curve), whereas for $s>0$ the cut must be between branches of the tree (red curve).

Near $s=0$, however, entanglement is high no matter how we cut the system. We verify this by plotting the minimum entanglement entropy over all possible bipartitions, without regard to locality. The resulting gray curves in Fig. 3b, for different partition sizes $L$, show a sharp peak in entanglement at $s=0$. Thus, at the crossover between the Archimedean and 2-adic geometries, there is no good notion of locality, and all spins are strongly coupled to one another.

Our non-local spin models generically exhibit quantum chaos. One indicator is that energy level spacings in the $s=0$ model at half filling $(N / 2$ magnons for $S=1 / 2)$ 
exhibit random-matrix statistics $[41,45,46]$. But is the highly connected model at $s=0$ a fast scrambler? To probe this question, we consider the out-of-time-order correlation function (OTOC) [19, 47-50]

$$
C(i, j ; t)=\left\langle\left|\left[S_{i}^{z}(0), S_{j}^{z}(t)\right]\right|^{2}\right\rangle / S^{2},
$$

where $S_{i, j}^{z}$ are local spin operators at sites $i, j$ and $S_{j}^{z}(t)=$ $e^{i H t} S_{j}^{z} e^{-i H t}$. In a typical fast scrambler, $C$ approaches its saturation value on a timescale $t_{*} \propto \log (N) / \lambda$, where $\lambda>0$ is a Lyapunov exponent quantifying the system's exponential sensitivity to perturbations.

In systems with local interactions, fast scrambling is precluded by the Lieb-Robinson bound [9, 51], which restricts $C \lesssim e^{-(d-v t)}$ to exponentially small values at distances $d=|i-j|$ outside a light cone with Lieb-Robinson velocity $v$, thereby preventing saturation of OTOCs until a time $t_{*} \propto N$. By contrast, known models for fast scrambling feature random all-to-all couplings $[52,53]$ and have no sense of spatial locality. Our model at $s=0$ offers an alternative route to fast scrambling: despite its effective light cone vs. graph distance $r_{i j}$ (Fig. 2a(iv)), the early-time growth of OTOCs is permitted to reach values $C \sim e^{-\left(r_{i j}-v t\right)} \sim 1 / N^{\alpha}[51]$ due to the logarithmic graph diameter $r_{\max } \approx \frac{1}{2} \log _{2}(N)$, where $\alpha$ is a constant of order unity and $v \propto J_{0} \log (N)$ because each spin has $\log (N)$ couplings. Subsequent Lyapunov growth $C \sim e^{\lambda t} / N^{\alpha}$ therefore allows OTOCs to reach saturation in a time $t_{*} \sim \alpha \log (N) / \lambda$.

A Lyapunov regime, however, is not guaranteed or even expected in a finite-size system with small local Hilbertspace dimension, e.g., at spin $S=1 / 2$ [54]. We thus first analyze the $s=0$ model semiclassically in the limit where each site contains a spin $S \gg 1$, which is natural to implement experimentally by letting each $\mathbf{S}_{i}$ represent the collective spin of an ensemble. We consider the averaged sensitivity

$$
C_{\mathrm{cl}}(i, j ; t)=\frac{1}{S^{2}}\left\langle\left(\frac{d S_{j}^{z}(t)}{d \phi_{i}}\right)^{2}\right\rangle,
$$

for a small initial rotation $\phi_{i}$ of spin $i$ about the $z$-axis, whose correspondence to the OTOC can be observed by replacing the commutator in Eq. (5) with a Poisson bracket $[55,56]$. We calculate the average in Eq. (6) for an ensemble in which each spin has a random initial orientation in the $x y$-plane, the classical limit of an infinite-temperature state at half filling.

The growth in sensitivity $C_{\mathrm{cl}}(t)$ generically exhibits two distinct regimes [20], visible in Fig. 4. The first is a rapid power-law growth $C_{\mathrm{cl}}(t) \propto\left(J_{0} t\right)^{2 r_{i j}}$ for $J_{0} t \lesssim 1$. A transition to exponential growth occurs for $J_{0} t \gtrsim 1$. Crucial to fast scrambling is that, by the time the exponential growth begins, the OTOCs have already reached values $C_{\mathrm{cl}} \sim 1 / N^{\alpha}$. We expect the subsequent exponential growth $C_{\mathrm{cl}}(t) \sim e^{\lambda t} / N^{\alpha}$ to yield a value $C_{\mathrm{cl}}\left(t_{*}\right) \sim 1$
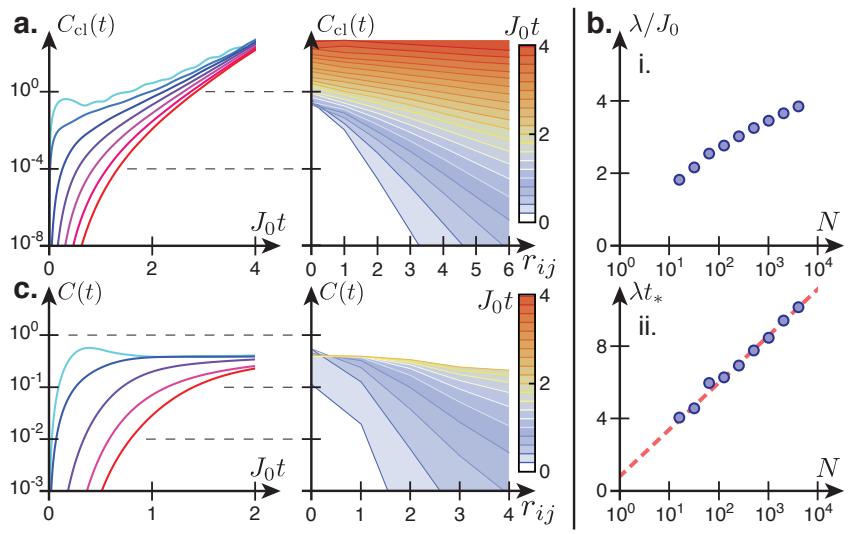

FIG. 4. Chaos and fast scrambling at $\mathbf{s}=\mathbf{0}$. (a) Average semiclassical sensitivity $C_{\mathrm{cl}}(t)$ for $N=4096$. (i) $C_{\mathrm{cl}}$ vs. time for graph distances $r_{i j}=0,1, \ldots 6$ (blue to red). (ii) Fixedtime contours of $C_{\mathrm{cl}}$ vs. $r_{i j}$, showing exponential decay of $C_{\mathrm{cl}}$ vs. $r_{i j}$ at early times. Contours are for $0.2 \leq J_{0} t \leq 4$ in increments of 0.2 . (b) Lyapunov exponent $\lambda / J_{0}$ (i) and scrambling time $\lambda t_{*}$ (ii) vs. $N$ (blue circles) with fit $\lambda t_{*}=$ $\alpha \log (N)+\beta$ (red dashed). (c) MPS calculations for $N=64$, $S=1 / 2$, and open boundary conditions.

at time $t_{*} \sim \alpha \log (N) / \lambda$. To verify this behavior, we first fit the exponential growth for a range of system sizes to obtain the dependence of the Lyapunov exponent $\lambda$ on $N$, and then evaluate the time $t_{*}$ to reach $C_{\mathrm{cl}}\left(t_{*}\right)=1$ in terms of $\lambda$ (Fig. 4b). Fitting the dependence of this semiclassical scrambling time on $N$ yields $\lambda t_{*}=\alpha \log (N)+\beta$, with $\alpha=1.1(1)$.

Our semiclassical analysis demonstrates that the correlations developing at early times $J_{0} t \lesssim 1$, before the onset of exponential growth, are crucial for enabling fast scrambling: the weakest correlations should be only algebraically small $\left(C \propto N^{-\alpha}\right)$ and not exponentially small in $N$. We now apply this insight to investigate whether the quantum model at $S=1 / 2$ can be a fast scrambler.

As the essence of fast scrambling is efficient spreading of information across an exponentially large Hilbert space, the process is intrinsically difficult to study numerically. Nevertheless, we calculate the early-time dynamics for $N=64$ sites at half filling using Matrix Product State (MPS) techniques [41, 57-61], for a system at infinite temperature with open boundary conditions. We see no Lyapunov regime, which is not surprising since there is no small parameter for $S=1 / 2$ at finite $N$ to prevent rapid saturation of the OTOC (Fig. 4c). However, Fig. 4c(ii) indicates early-time correlations that fall off exponentially with graph distance $r_{i j}$, consistent with correlations across all sites that are algebraically rather than exponentially small in $N$ - the aforementioned necessary condition for fast scrambling.

Our results indicate that fast scrambling is accessible in sparsely coupled models without disorder, and might generalize to a wider range of coupling patterns in which 
the graph diameter grows logarithmically with system size. Near-term cavity-QED experiments offer promise for observing the logarithmic timescale for spin transport at $s=0$, as well as the linear-to-treelike transition. In the large- $S$ regime, where each site contains an atomic ensemble, experiments will benefit from a collective enhancement in the coherence of interactions. Ultimately, implementations of the spin-1/2 model-for sufficiently strong atom-light coupling [41, 62] — could test for fast scrambling $[19,41,49,50]$ at large $N \sim 10^{3}$ in the quantum regime.

Complementarily, future theoretical work may investigate whether a Lyapunov regime can emerge in the spin$1 / 2$ quantum model through a coarse-graining procedure that exploits the self-similarity of the coupling pattern. Measures of entanglement throughout the linearto-treelike transition also merit further study, and may enable a more explicit connection to holography via entanglement wedges or tensor networks [22, 63-68]. Future work may also explore prospects for harnessing the rapid and deterministic generation of entanglement for quantum information processing.

This work was supported by the DOE Office of Science, Office of High Energy Physics. MS-S acknowledges support from the Research Corporation Cottrell Scholar Program and the NSF. SSG acknowledges support from the Simons Foundation. AJD, AB, and TH acknowledge support from the EPSRC Programme Grant DesOEQ (EP/P009565/1), and by the EOARD via AFOSR grant number FA9550-18-1-0064. We additionally acknowledge support from the NSF GRFP (EJD and GB) and Hertz Foundation (EJD), as well as support for international exchanges from SU2P. We thank Xiangyu Cao, Sean Hartnoll, Patrick Hayden, and Steve Shenker for helpful discussions.

[1] Y. Sekino and L. Susskind, J. High Energy Phys. 2008, 065 (2008).

[2] P. Hayden and J. Preskill, J. High Energy Phys. 2007, 120 (2007).

[3] J. Maldacena, S. H. Shenker, and D. Stanford, J. High Energy Phys. 2016, 106 (2016).

[4] J. Preskill, (2016), arXiv:1604.07450 [quant-ph].

[5] M. Gärttner, J. G. Bohnet, A. Safavi-Naini, M. L. Wall, J. J. Bollinger, and A. M. Rey, Nat. Phys. 13, 781 (2017).

[6] J. Li, R. Fan, H. Wang, B. Ye, B. Zeng, H. Zhai, X. Peng, and J. Du, Phys. Rev. X 7, 031011 (2017).

[7] K. X. Wei, C. Ramanathan, and P. Cappellaro, Phys. Rev. Lett. 120, 070501 (2018).

[8] K. A. Landsman, C. Figgatt, T. Schuster, N. M. Linke, B. Yoshida, N. Y. Yao, and C. Monroe, Nature 567, 61 (2019).

[9] E. H. Lieb and D. W. Robinson, Comm. Math. Phys. 28, 251 (1972).

[10] M. Cheneau, P. Barmettler, D. Poletti, M. Endres,
P. Schauß, T. Fukuhara, C. Gross, I. Bloch, C. Kollath, and S. Kuhr, Nature 481, 484 (2012).

[11] P. Richerme, Z.-X. Gong, A. Lee, C. Senko, J. Smith, M. Foss-Feig, S. Michalakis, A. V. Gorshkov, and C. Monroe, Nature 511, 198 (2014).

[12] S. Bravyi, M. B. Hastings, and F. Verstraete, Phys. Rev. Lett. 97, 050401 (2006).

[13] M. B. Hastings and T. Koma, Commun. Math. Phys. 265, 781 (2006).

[14] M. Foss-Feig, Z.-X. Gong, C. W. Clark, and A. V. Gorshkov, Phys. Rev. Lett. 114, 157201 (2015).

[15] D. V. Else, F. Machado, C. Nayak, and N. Y. Yao, (2018), arXiv:1809.06369.

[16] I. Danshita, M. Hanada, and M. Tezuka, Prog. Theor. Exp. Phys. 2017, $083 \mathrm{I} 01$ (2017).

[17] A. Chen, R. Ilan, F. de Juan, D. Pikulin, and M. Franz, Phys. Rev. Lett. 121, 036403 (2018).

[18] A. Chew, A. Essin, and J. Alicea, Phys. Rev. B 96, 121119(R) (2017).

[19] B. Swingle, G. Bentsen, M. Schleier-Smith, and P. Hayden, Phys. Rev. A 94, 040302(R) (2016).

[20] J. Marino and A. Rey, Physical Review A 99, 051803(R) (2019).

[21] S. S. Gubser, J. Knaute, S. Parikh, A. Samberg, and P. Witaszczyk, Commun. Math. Phys. 352, 1019 (2017).

[22] M. Heydeman, M. Marcolli, I. Saberi, and B. Stoica, (2016), arXiv:1605.07639 [hep-th].

[23] J. L. F. Barbón and J. M. Magán, J. High Energy Phys. 2012, 16 (2012).

[24] J. L. F. Barbón and J. M. Magán, J. High Energy Phys. 2013, 163 (2013).

[25] C.-L. Hung, A. González-Tudela, J. I. Cirac, and H. Kimble, Proc. Natl. Acad. Sci. U.S.A. 113, E4946 (2016).

[26] S. Gopalakrishnan, B. L. Lev, and P. M. Goldbart, Nature Physics 5, 845 (2009).

[27] I. D. Leroux, M. H. Schleier-Smith, and V. Vuletic, Phys. Rev. Lett. 104, 073602 (2010).

[28] P. Strack and S. Sachdev, Phys. Rev. Lett. 107, 277202 (2011).

[29] O. Hosten, R. Krishnakumar, N. J. Engelsen, and M. A. Kasevich, Science 352, 1552 (2016).

[30] S. Welte, B. Hacker, S. Daiss, S. Ritter, and G. Rempe, Phys. Rev. X 8, 011018 (2018).

[31] E. J. Davis, G. Bentsen, L. Homeier, T. Li, and M. H. Schleier-Smith, Phys. Rev. Lett. 122, 010405 (2019).

[32] R. M. Kroeze, Y. Guo, V. D. Vaidya, J. Keeling, and B. L. Lev, Phys. Rev. Lett. 121, 163601 (2018).

[33] M. A. Norcia, R. J. Lewis-Swan, J. R. Cline, B. Zhu, A. M. Rey, and J. K. Thompson, Science 361, 259 (2018).

[34] M. Landini, N. Dogra, K. Kroeger, L. Hruby, T. Donner, and T. Esslinger, Phys. Rev. Lett. 120, 223602 (2018).

[35] B. Braverman, A. Kawasaki, E. Pedrozo-Peñafiel, S. Colombo, C. Shu, Z. Li, E. Mendez, M. Yamoah, L. Salvi, D. Akamatsu, Y. Xiao, and V. Vuletic, (2019), arXiv:1901.10499.

[36] F. Borjans, X. G. Croot, X. Mi, M. J. Gullans, and J. R. Petta, (2019), arXiv:1905.00776.

[37] F. Mivehvar, H. Ritsch, and F. Piazza, Phys. Rev. Lett. 122, 113603 (2019).

[38] Related fractal behavior was recently found in a simplified statistical mechanical model with sparse couplings [44] of essentially the same form as in Eq. (2). 
[39] The tree appears to break translation invariance, but in fact it does not: $d_{\text {tree }}(i+1, j+1)=d_{\text {tree }}(i, j)$.

[40] We thank P. Hayden for discussions on this point.

[41] See Supplemental Material at [URL will be inserted by publisher] for supporting derivations and additional experimental details, including Refs. [42-43].

[42] J. J. García-Ripoll, New J. Phys. 8, 305 (2006).

[43] S. Paeckel, T. Köhler, A. Swoboda, S. R. Manmana, U. Schollwöck, and C. Hubig, (2019), arXiv:1901.05824.

[44] S. S. Gubser, C. Jepsen, Z. Ji, and B. Trundy, Phys. Rev. D 98, 045009 (2018).

[45] P. Weinberg and M. Bukov, SciPost Phys. 2, 003 (2017).

[46] S. Wimberger, Nonlinear Dynamics and Quantum Chaos (Springer International Publishing, 2014).

[47] A. I. Larkin and Y. N. Ovchinnikov, Soviet Journal of Experimental and Theoretical Physics 28, 1200 (1969).

[48] S. H. Shenker and D. Stanford, J. High Energy Phys. 2014 (2014), 10.1007/JHEP03(2014)067.

[49] B. Swingle and N. Yunger Halpern, Phys. Rev. A 97, 062113 (2018).

[50] Y.-L. Zhang, Y. Huang, and X. Chen, Phys. Rev. B 99, 014303 (2019).

[51] G. Bentsen, Y. Gu, and A. Lucas, Proc. Natl. Acad. Sci. U.S.A. 116, 6689 (2019).

[52] N. Lashkari, D. Stanford, M. Hastings, T. Osborne, and P. Hayden, J. High Energy Phys. 2013, 22 (2013).

[53] J. Maldacena and D. Stanford, Phys. Rev. D 94, 106002 (2016).
[54] V. Khemani, D. A. Huse, and A. Nahum, Phys. Rev. B 98, 144304 (2018).

[55] A. Polkovnikov, Ann. Phys. (N. Y.) 325, 1790 (2010).

[56] J. S. Cotler, D. Ding, and G. R. Penington, Ann. Phys. (N. Y.) 396, 318 (2018).

[57] U. Schollwoeck, Ann. Phys. (N. Y.) 326, 96 (2011).

[58] J. Haegeman, J. I. Cirac, T. J. Osborne, I. Pižorn, H. Verschelde, and F. Verstraete, Phys. Rev. Lett. 107, 070601 (2011).

[59] T. Koffel, M. Lewenstein, and L. Tagliacozzo, Phys. Rev. Lett. 109, 267203 (2012).

[60] J. Haegeman, T. J. Osborne, and F. Verstraete, Phys. Rev. B 88, 075133 (2013).

[61] J. Haegeman, C. Lubich, I. Oseledets, B. Vandereycken, and F. Verstraete, Phys. Rev. B 94, 165116 (2016).

[62] Y. Colombe, T. Steinmetz, G. Dubois, F. Linke, D. Hunger, and J. Reichel, Nature 450, 272 (2007).

[63] B. Swingle, (2012), arXiv:1209.3304.

[64] F. Pastawski, B. Yoshida, D. Harlow, and J. Preskill, J. High Energy Phys. 2015, 149 (2015).

[65] A. Bhattacharyya, L.-Y. Hung, Y. Lei, and W. Li, J. High Energy Phys. 2018, 139 (2018).

[66] M. Heydeman, M. Marcolli, S. Parikh, and I. Saberi, (2018), arXiv:1812.04057 [hep-th].

[67] G. Vidal, Phys. Rev. Lett. 101, 110501 (2008).

[68] Y.-Y. Shi, L.-M. Duan, and G. Vidal, Phys. Rev. A 74, 022320 (2006). 